\title{
Towards a Transdisciplinary Approach to Systemic Risk Detection
}

\author{
Mark WEVER ${ }^{\mathrm{a}, 1}$, Nel WOGNUM ${ }^{\mathrm{b}}$, MUNIR SHAH ${ }^{\mathrm{a}}$, Niall O'LEARY ${ }^{\mathrm{c}}$ and George \\ ONOFREI $^{\mathrm{d}}$ \\ a AgResearch, Lincoln, New Zealand \\ ${ }^{b}$ TU Delft, ATO Group, Delft, The Netherlands \\ ${ }^{c}$ Cork Institute of Technology, Ireland \\ ${ }^{d}$ Letterkenny Institute of Technology, Ireland
}

\begin{abstract}
Systemic risks are potentially harmful events, that could severely disrupt an entire industry or economy. Examples include the bankruptcy of keystone companies and biosecurity incursions. According to the United Nations, detecting and managing systemic risk represents one of the main challenges of the $21 \mathrm{st}$ century. Due to increasing complexity and interconnectedness of today's socialtechnological-biophysical systems, stakeholders relying on individual disciplines to systemic risk detection, or a combination of disciplines that are not well coordinated, will fail to promptly identify key early warning signals of threats. Our paper argues that transdisciplinary approaches are required to make comprehensive and integrative assessments of complex systems. To support stakeholders undertaking such assessments, we propose a framework that will assist them in: (1) better understanding their system and the risks to which it is exposed; (2) selecting complementary disciplines, theories and methods that are relevant to the system and risks in question; and (3) integrating knowledge from these different disciplines to detect a wide range of early warning signals of systemic risk. The framework can be used as a foundation to build transdisciplinary approaches to risk detection.
\end{abstract}

Keywords. Transdisciplinary, systemic risk, risk detection, early warning systems

\section{Introduction}

Our social, technological and biological systems are becoming ever more integrated through trade, travel and communication. This makes these systems more complex and dynamic, preventing equilibria from forming and establishing, and so makes them increasingly unstable. In such systems, it is hard to identify and mitigate systemic fragilities and risk. According to the United Nations (UN), the management of systemic risk is expected to be the main global challenge of the next decades [1].

Over the last half a century or so, researchers, analysts, regulators and other stakeholders $^{2}$ have started to build Early Warning Systems (EWSs) for detecting systemic risks. EWSs have been developed and deployed for the detection of a wide range of risks, including: missile attacks, virus outbreaks, earthquakes, and financial

\footnotetext{
${ }^{1}$ Corresponding author, Mail: mark.wever@agresearch.co.nz

2 Stakeholders refers to the actors that have an interest in a system's functioning or success. This includes the actors directly involved in a system (e.g., farmers in the food production system), but also some external actors (e.g., consumers of food products).
} 
crises [2][3][4]. Many of these EWSs have been constructed with a narrow focus in mind - dedicated to spotting warning signals in specific sub-systems and rely upon a limited range of indicators, tools and science disciplines. However, due to the increase in complexity and interconnectedness of our systems, such narrow-focused approaches to systemic risk detection will likely either miss key warning signals of threats or not anticipate their impact correctly [5][6].

To be able to understand complex social-technological-biophysical systems, and what types of risks lurk within them, we argue that insights from a wide range of science disciplines and stakeholders are necessary, and a transdisciplinary approach is required in order to make these different disciplines and stakeholders work well together. Narrowfocused approaches will be unable to detect enough systemic vulnerabilities in modern day complex systems [1]. Therefore, there is a need to enhance the ability to look at a system from different angles, reducing blind spots and developing a variety of scenarios about how a system may evolve.

While some integrated research approaches to the study of systemic risk have been undertaken [7][8], work in this area is still at an early stage. Especially, a robust framework for linking and integrating knowledge from different disciplines and stakeholders in a coherent fashion is lacking. Thus, developing such a framework is critical to bringing research in this area forward. Failing to synchronize and combinations of non-complementary disciplines, theories, methods, and models will not help researchers and stakeholders make comprehensive assessments of complex systems.

In this paper, we present a framework for developing transdisciplinary approaches to systemic risk detection. The framework is meant to assist researchers and stakeholders in: (1) better understanding their system and the risks to which it is exposed; (2) selecting complementary disciplines, theories and methods that are relevant to the system and risks in question; and (3) integrating knowledge from these different disciplines to detect a wide range of early warning signals of systemic risk. The remainder of the paper is organized as follows. Section 1 outlines the basics of systemic risk. Section 2 gives a brief overview of the theories and methods used to detect systemic risk based on a targeted literature review. Articles for the review were selected based on the scalability of the discussed theories and methods to different types of context and risks. Section 3 discusses some of the difficulties that arise in systemic risk detection and explains how transdisciplinary approaches can help to overcome these difficulties. Section 4 presents a framework for developing such approaches. The final section concludes the paper.

\section{Systemic risk}

Systemic risks are potentially harmful events that could severely disrupt or even do unrepairable damage to a network if they were to occur [5][9]. Systemic risks are often triggered by failures at key nodes within the network, which can cause rippling effects across the remainder of the network [10]. For example, in financial networks, the bankruptcy of a large and well-connected bank, may cause other banks to go bankrupt as well.

Systemic risks tend to arise predominantly in complex networks [7]. The relationships and interactions between the numerous elements making up such networks (e.g., cities, markets, coral reefs) are often difficult to comprehend. This complexity is accentuated by their dynamism, which may make these relationships also unstable [11][5]. As a result, researchers and stakeholders struggle to understand what types of 
risks may be hiding within the network and find it difficult to predict how the network may respond to external events or threats.

Systemic risks can be distinguished alongside various dimensions. First, is the timeinterval between the first signal of an upcoming event and its impact. The onset of a systemic event can be very rapid or a train wreck in slow motion. Covid 19 is a good example of the former and climate change exemplifies the latter. For an EWSs to be of value, intervention needs to be possible between the time a signal is detected, and the time the event occurs or makes its impact (e.g., a tsunami alert in case of an earthquake).

Second, systemic risks can be classified based on the strength of the signal. Signals can be non-existent, weak, or strong. In general, signals increase in strength as a crisis draws closer. For example, signals that something was wrong in the financial services system became clearer over time in the lead-up to the 2008 sub-prime mortgage crisis. EWSs are mainly valuable in the context of picking up weak signals early, so that stakeholders have more options to mitigate a risk or prepare for its adverse consequences.

Third and last, systemic risks can be differentiated based on their genesis: internally or externally generated. An internal risk is caused by factors endogenous to a system (e.g., mismanagement at a key company), while an external risk is caused by exogenous factors (e.g., an energy crisis because of global political tension). However, it can be difficult to distinguish between internal and external risks, as exogenous factors often interact with endogenous factors (e.g., as when the effect of mismanagement at a key company become more glaring and pronounced because a global recession is ongoing). In today's interconnected world, for an EWS to be of value in making a comprehensive assessments of threats to a system, both internal and external risks need to be considered.

\section{Systemic risk detection: theories and methods}

We briefly discuss some of the key theories and methods/tools used to detect systemic risk. "Detection" refers to the process of: (1) identifying a risk; (2) assessing its likely impact; and (3) communicating this assessment to analysts. A theory or tool may cover the whole process of risk detection, or just one or two components of it.

\subsection{Examples of theories}

A wide range of theoretical perspectives is used to study systemic risk [7][11]. Due to space limitations, we will focus on two relatively widely used frameworks: system theory and network theory. As both theories have their limitations as stand-alone frameworks, researchers and stakeholders should consider using multiple theories when studying systemic risks, including other perspectives.

System's theory is used to identify and assess the processes that cause a system's structure to change and evolve. Systems theory focuses on the self-organizing capabilities of complex systems or structures [12][7][13]. How does a complex system evolve and adapt over time? What is the influence of the system's initial structure or conditions on the system's evolutionary path? How do a system's constituent components interactions influence this process? What feedback loops keep the system on a relatively predictable path? What are the interactions and feedback loops that make a system go 'off-track'?

System theory is foundational for systemic risk analysis but has a few limitations. Firstly, the theory gives limited guidance to researcher on the specific aspects of the 
system that they should focus on. Questions, such as how the boundaries of the system should be determined or how key interdependencies and feed-back loops of a system can be identified a priori, remain insufficiently addressed. This limits its usefulness for model variable selection. For example, if a system's boundaries, or interactions with neighboring systems are not well-understood, externally generated systemic risks may be missed. Secondly, many approaches based on system theory are preoccupied with bottom-up changes to the system and often insufficiently consider top-down driven changes. As a result, certain risks may be missed, such as rapid on-set events arising from failure at central institutions.

Network theory describes a system's structure in terms of relationships between the elements [11]. Activity within the system, such as the sharing of information between human agents or the spread of a viral disease, is considered to be enabled or constrained by that structure [14]. For example, all else being equal, a viral disease will spread more easily within dense networks than in sparsely connected networks with largely selfcontained, isolated hubs of agents.

Like systems theory, network theory is central to understanding and analysing systemic risk. However, also like systems theory, it has a couple of limitations. For example, many approaches that rely on network theory insufficiently consider the multilayered nature of complex systems, which have a range of different types of overlapping networks. Researchers that don't take this property of systems into accounts are likely to make erroneous conclusions. For example, the complexity of the economic ties between agents in the financial system has increased drastically over the last three decades. But, this complexity has been for a large part enabled by an accompanying increase in the complexity of the communications network supporting those economic ties. Without taking this into account, assessments about the stability of the current structure of the financial system are going to be ill-informed.

\subsection{Trends in systemic risk detection methods and tools}

Multi-agent simulations, sentiment analysis, graph theory, network modelling, Bayesian networks, and systems dynamics are some of the key methods, tools and techniques that have been applied to the study of systemic risk [15][9][16][17]. These techniques have been used at various levels of system analysis, including to simulate the micro-level behaviour of market participants, to identify critical clusters within networks, to simulate interaction effects between different sectors of the economy, and more generally to anticipate positive feedback loops and non-linear transformations in the system.

Furthermore, researchers have started to develop hybrid approaches, whereby a combination of different types of techniques is applied to the study of systemic risk. For example, Paulin et al. combine an agent-based model with network modelling to study the conditions that lead to "flash crash" events in financial markets [18]. The Bank of England uses a collection of models to cross-check projections made based on a general equilibrium model [19]. Bradhurst et al. combine a population-based model with an agent-based model to simulate the spread of livestock diseases [20].

The basic logic behind the trend towards hybrid approaches is that while individual techniques can be useful for studying individual parts of the system, or to obtain a narrow perspective on the functioning of the system as a whole, they need to be combined with other tools and techniques to address the limitations of each individual tool and to obtain a more comprehensive view of the system. Hybrid approaches address the need for more integrated, comprehensive approaches to the study of complex systems. 
However, they are at an early stage of development, often only loosely linking a limited number of techniques from closely related disciplines. At present, such approaches are unable to capture all key interdependencies that exist amongst system components.

\section{Systemic risk detection challenges}

Section 3.1 discusses the difficulties in information- access and processing that riskmanagers face in their systemic risk detection attempts and argues that inter- or transdisciplinary approaches are required to help them deal with these difficulties. Section 3.2 discusses some of the conflicts of interests and biases that a wider group of stakeholders face in dealing with complex systems and explains how they tend to lead to under-investment in risk management activities. More active stakeholder involvement in risk-management decision making could help to address this. Transdisciplinary approaches are especially suitable for enabling such stakeholder involvement.

\section{1. "Technical" difficulties in systemic risk-management}

Systemic risk management activities are usually carried out by regulatory agencies representing the interests of external stakeholders (e.g., a food safety regulator), through collective institutions that represent a wider range of agents (e.g., an industry board), as well as by the larger agents within the system (e.g., a large slaughterhouse that keeps track of food safety issues across the meat industry). Such risk-managers usually face various difficulties in identifying, assessing, and managing systemic risk.

Firstly, information about the system may be missing or out-of-date. For example, assessments of financial systemic risk based on the balance sheet data of large financial institutions maybe a good reflection of the state of the system at a particular point in time. However, the system may have already shifted towards a new state by the time this data becomes available to analysts. Secondly, even if key information is promptly available, it may be difficult to process it in a timely manner. For example, thousands of pages would have had to been read to fully assess the risks of some of the securities that helped cause the subprime crisis [11]. Thirdly, the state of the system that increases the likelihood of a systemic risk occurring may not be fully transparent to (external) stakeholders. For example, many food systems are made up of numerous actors, operating in a wide range of countries. This makes it complicated to promptly detect fraudulent activities, such as those that led to the European horse meat scandal. Fourthly, the consequences of systemic events are generally difficult to estimate ex-ante, even in the case of well-known and widely assessed risks (e.g., climate change).

Ultimately, the above-mentioned difficulties tie back to the problem of anticipating the evolutionary path of complex systems based on incomplete information and limited information processing abilities. With complex systems, often a plurality of opaque, future scenarios is plausible [6]. To be able to anticipate as many of these scenarios as is possible, it is necessary to assess the system from multiple angles, disciplines, and techniques. This helps to hedge the risk that any individual technique will fail to promptly identify a warning signal of risk. However, to detect systemic risk requires not only that a wider range of disciplines study the system, but also smarter approaches for making these different disciplines work in sync. Individual disciplines cannot offer stakeholders the diversity of models and perspectives that is necessary to untangle a complex system, while a combination of disciplines that are not well- 
coordinated (a multidisciplinary approach) will not help stakeholders to link or integrate these perspectives into a well-rounded picture of the system.

\subsection{Disincentives, biases, and under-investment in systemic risk management}

In addition to imperfect insight into the system's possible evolutionary paths, riskmanagers and other stakeholders also often face various disincentives and biases against undertaking sufficient risk-management activities [21][22] [13], including:

- Many systemic risks are rare events (e.g., a global financial crisis), appear to be in the distant future (e.g., job losses as a result of advances in AI and robotics), or are "slow-burner" events (e.g., climate change). Companies due to shareholders and politicians due to voters usually take a more shorter-term view. Therefore, agents and external stakeholders often defer adequately preparing for systemic risk.

- Consumers and citizens more generally, often take a short-term view as well. This is in part due to reasoning errors where future probable costs are overly discounted.

- Conflicts of interests may also exist. For example, an older citizen may be less willing to commit resources to preventing distant future or slow-burner events than a younger citizen (who will bear the brunt of these events).

- Humans evolved in small, technological undeveloped groups and communities. Our ability to intuitively understand complex systems is thus naturally poor.

Interdisciplinary approaches deal with well-defined scientific problems. The conflicts of interests between stakeholders in complex systems mentioned above suggest that interdisciplinary approaches will be insufficient to deal with many systemic risks. Combining interdisciplinary research with multi-stakeholder discourse, i.e. transdisciplinary approaches are thus required [23]. Transdisciplinary research deals with problems that are less clear (e.g., how to anticipate black swans). They also have more explicitly normative components (e.g., how to balance inter-temporal trade-offs) [23]. Such problems are common in complex systems, where many different actors operate with competing interests [24].

\section{Towards a transdisciplinary approach}

Our framework for developing transdisciplinary approaches to systemic risk detection is visualized in Figure 1. The framework is based both on the characteristics of complex systems and systemic risks, as well as on our own experiences in developing an approach to detecting biosecurity-related systemic risks in New Zealand.

The framework consists of three iterative loops which feed into each other. In the integrated development loop, the scientific disciplines and stakeholders that are needed to assess the system are selected and an approach for working together is developed. In the knowledge development loop, the researchers and stakeholders use this approach to co-develop knowledge about how to assess the risks to which the system is exposed. This knowledge is then applied to assess the system in question in the system's analysis loop. During this analysis loop, new information and learnings will be obtained about the system, as well as feedback about the suitability of the developed approach for assessing it. This feedback is then used by the researchers and stakeholders to expand and build on their knowledge about the system and how to best assess it. This modified approach can then be applied to make new system assessments, from which again 
additional information will be obtained about the suitability of the developed approach for assessing it. This should lead to a continuous learning loop in which the approach and tools used to assess the system - as well as the ways in which people work together to create and modify these tools - are regularly questioned, evaluated and improved, as knowledge is never taken for granted about quickly changing systems.

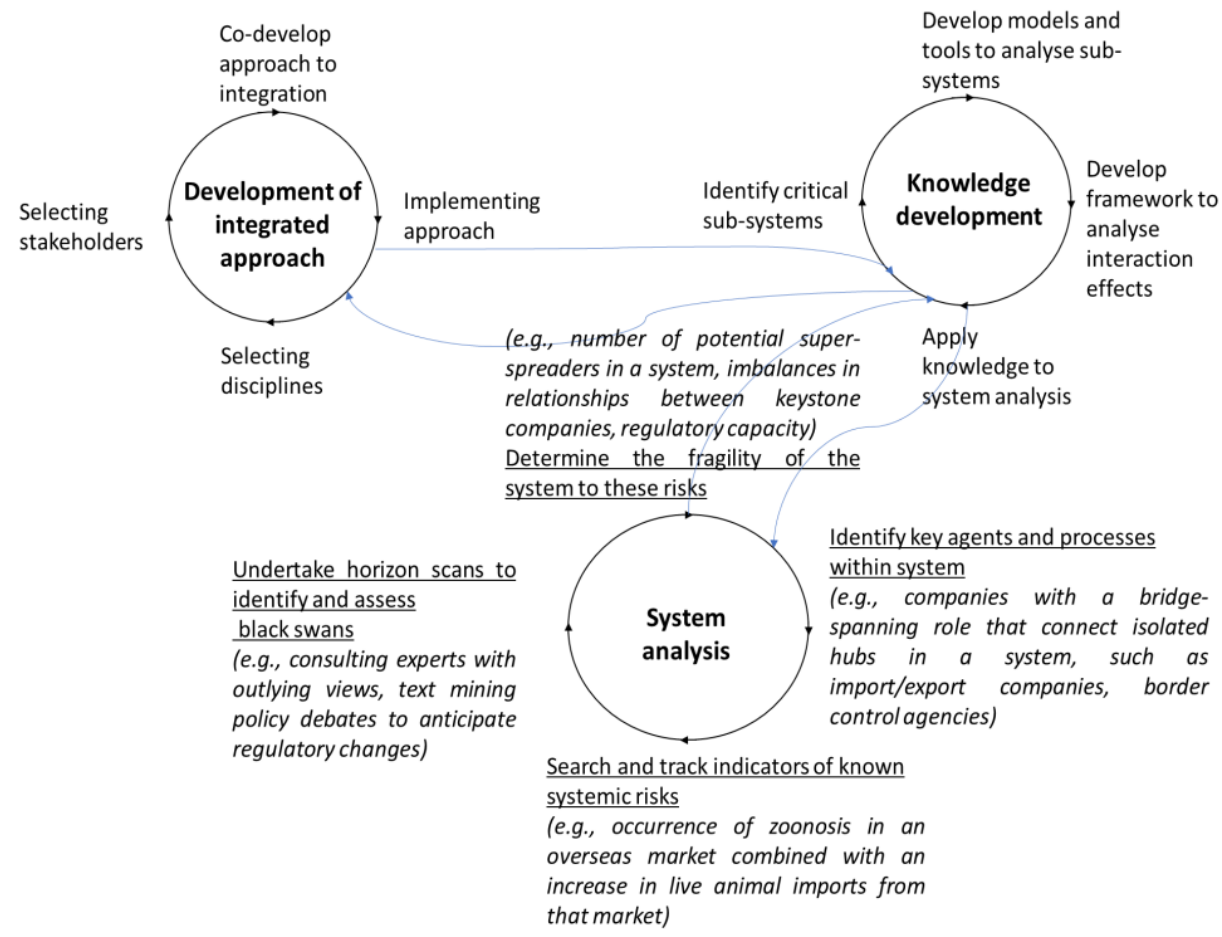

Figure 1. Framework for developing TD approaches to systemic risk detection.

The integrated development loop consists of 4 phases: (1), selecting disciplines; (2), selecting stakeholders; (3), developing an approach to working together; (4), implementation. The initial selection of disciplines and stakeholders will most likely be intuitive, as in many transdisciplinary research projects. However, over time, after the first loop of the integrated development phase is completed, the selection should become more structured and driven by gaps in the approach used to analyse the system. Cognitive diversity is important to the process of systemic risk detection (e.g., see [25]) and it therefore pays to be inclusive during the selection process. In the next phase, researchers and stakeholders have to build consensus and capacity about how to work together and agree upon strategies and methods for integrating knowledge. To be able to deal with system complexity, it is useful to split-up the larger project team into several smaller teams, each responsible for developing a model of a sub-component of the system [26]. Furthermore, it is also necessary to create teams responsible for identifying and studying interaction effects between the various sub-components. To ensure that a plurality of perspectives on the state of the system will be realized, which is important as in complex systems a plurality of different types of futures is often equally likely [6], multiple teams will have to be tasked with assessing the same sub-systems, but through different approaches, lenses and with a different make-up in terms of involved disciplines and 
stakeholders. Additionally, to help ensure such a plurality of perspectives, interaction between teams analysing the same sub-components of the system needs to be limited until after the system analysis phase (after which mutual learnings can take place about what worked, and what didn't). The teams responsible for integrating results from the different sub-system analysis will have to devise methods for aggregating results, either by combining the results from different types of assessments, or by establishing a hierarchy in the quality of the assessments [27]. A hierarchical approach is preferred in the context of systemic risk, as the underlying assessments are preserved more directly, helping to maintain a plurality of perspectives. Finally, the developed approach is put into practice, first by co-developing the knowledge required to assess the system, and subsequently by applying that knowledge to assess the system.

In the first phase of the knowledge development loop, the system is broken down conceptually into sub-components, which can be more easily modelled and analysed [26]. The focus here should be on those sub-systems that put the survival of the system at risk if they fail to function properly. For example, in the context of economic systems, these are often industries that are both large and that perform a critical service to the other industries within the system [28]. In this phase, also key sub-systems in neighbouring systems should be identified. These are sub-systems that interact with the focal system in question, and which may therefore be a source of systemic risk. In the second phase, models and tools are developed that will help the teams identify and assess early warning signals of potential failure points within the critical sub-systems, both within and outside of the focal system. For example, in the context of biosecurity risks, an internal sub-system may be a country's border biosecurity institutions, while an external sub-system may be the border security institutions of the country's main trading partners. In the third phase, a framework is developed to help the teams look for interaction effects between the different sub-systems, a necessary step when a problem is broken down into constituent components [26]. The theories discussed in section two form useful starting points for developing such a framework. This framework should help the teams to create a "system of models", whereby linkages are established between the sub-system level models and tools. For example, as when the output of a model used to assess "sub-system A" is used as input in a model to assess "sub-system B". This will require the development of tools that help to coordinate the outputs of the sub-system level analyses. Once the framework and tools are developed, the teams can put their knowledge to the test.

In the first phase of the system's analysis loop, the objective is to identify or confirm for each of the sub-systems, as well as for the system as a whole, which actors (e.g., companies, regulators ) and processes (e.g., inter-bank lending) are key to the (sub-)system's functioning. While many actors and processes will have already been identified in the knowledge development phase, it is essential to undertake a dedicated study to ensure that no critical agents or processes are missed, as they are a key source of systemic risk, both of well-known risks and black swans. Subsequently, the teams can use their framework and tools to scan and monitor for early warning signals of both categories of systemic risks [5], as well as for assessing the fragility of the system against these risks [7]. These three phases are tightly interwoven, as the extent to which an event is a risk depends in large part upon the system's fragility against the risk. It is here that the benefits of taking a transdisciplinary approach becomes apparent [1], as people from a wide range of disciplines working together in an integrated fashion will be more likely to identify risks [29]. 
"Non-transdisciplinary" approaches would miss more risks. Firstly, monodisciplinary approaches assess the system from a single discipline and thus view the system from a limited range of perspectives. Secondly, multi-disciplinary approaches insufficiently integrate the insights from different disciplines and thus will miss more interaction effects between processes or system sub-components. Finally, interdisciplinary approaches fail to integrate "ground truths" from stakeholders and so are likely to have developed models of the system that contain unrealistic assumptions [30].

Key to the final three phases of the system's analysis loop is that the mechanisms to limit group think that were developed in the "integrated approach development loop" are well-functioning. This is to ensure that the assessments of analysts with outlying views about the likelihood or impact of a certain risk are not easily dismissed by the majority opinion. For black swans, which by definition are unexpected events, outlying views will be extremely valuable in picking up early warning signals.

\section{Discussion and conclusion}

Systemic risks arise predominantly in complex, dynamic systems. Compared to simpler systems, complex systems have a wider set of possible futures, which are more difficult to foresee, and for which it is also more difficult to predict the likelihood of their occurrence. To be able to correctly anticipate and assess as many of these futures as possible, and thus pick up as many warning signals of systemic risks as possible, transdisciplinary approaches to risk detection are necessary.

In the present paper, we have presented a framework that can help researchers and stakeholders co-develop transdisciplinary approaches to systemic risk detection. Key to the successful implementation of the framework is the creation of feedback mechanisms and a culture of continuous learning. Researchers and stakeholders should continuously question, evaluate, and improve the approach and tools used to assess the system, as well as their ways of working together to create or modify these tools. This is essential in the context of complex, dynamic systems, where knowledge quickly can become outdated. Taking an ever-evolving approach to the analysis of complex systems should lead to more competent approaches to systemic risk detection.

\section{References}

[1] M. Jahn, F. Gaupp and M. Obersteiner, M. Assessing and analysing systemic risks: mapping the topology of risk through time (Chapter 2.1). In: Global Assessment Report on Disaster Risk Reduction (GAR). United Nations Office for Disaster Risk Reduction (UNDRR), Geneva, 2019, pp. 37-47.

[2] D. Gramlich, G. Miller, M.V. Oet, and S.J. Ong, Early warning systems for systemic banking risk. Banks and Bank Systems, 2010, Vol. 5, pp. 199-211.

[3] F. Mostashari, M. Kulldorff, J.J. Hartman, J. R. Miller And V. Kulasekera, Dead bird clusters as an early warning system for West Nile virus activity. Emerging infectious diseases, 2003, Vol. 9, pp. 641-646.

[4] M. Erdik, Y. Fahjan, O. Ozel, H. Alcik, A. Mert and M. GUL, Istanbul Earthquake Rapid Response and the Early Warning System. Bulletin of Earthquake Engineering, 2003, Vol. 1, pp. 157-163.

[5] N.N., IRGC Guidelines for the governance of systemic risks: In systems and organizations in the context of transitions. International Risk Governance Center, Lausanne, 2018.

[6] W.E. Walker, V.A.W.J. Marchau and D. Swanson, Addressing deep uncertainty using adaptive policies: Introduction to section 2. Technological Forecasting and Social Change, 2010, Vol. 77, pp. 917-923.

[7] J. Colchester and F. Filia, Financial complexity and nonlinear dynamics, 2018, Complexity Labs and Fasanara Capital, https://www.fasanaracapital.com/financial-complexity-and-nonlinear-, Accessed July, 32021.

[8] S. O'Halloran and N. Nowaczyk, An artificial intelligence approach to regulating systemic risk, Frontiers in Artificial Intelligence, 2019, https://doi.org/10.3389/frai.2019.00007. 
[9] G. Kou, X. Chao, Y. Peng, F.E. Alsaadi and E. Herrera-Viedma, Machine Learning Methods For Systemic Risk Analysis In Financial Sectors, Tech. and Econ. Dev. of Econ., 2019, Vol. 25, pp. 716-742.

[10] V. Sasidevan and N. Bertschinger, Systemic Risk: Fire-Walling Financial Systems Using Network-Based Approaches. In: A.S. Chakrabarti et al. (eds.) Network Theory and Agent-Based Modeling in Economics and Finance, Springer Nature Switzerland, 2019, pp. 313-330.

[11] A. Haldane, Rethinking the financial network. Amsterdam: Speech delivered at the Financial Student Association, 2009.

[12] D.H. Meadows, Thinking in Systems, Chelsea Green, Vermont, 2008.

[13] U. Wilensky and W. Rand, Modeling Natural, Social, and Engineered Complex Systems with NetLogo, MIT Press, Cambridge, 2015.

[14] M.O. Jackson and D. López-Pintado, Diffusion and contagion in networks with heterogeneous agents and homophily. Network Science, 2013, Vol. 1, pp. 49-67.

[15] R. Ojha, A. Ghadge, M.K. Tiwari and U. Bititci, Bayesian network modelling for supply chain risk propagation. International Journal of Production Research, 2018, Vol. 56, pp. 5795-5819.

[16] S.M. Guerra, T.C. Silva, B.M. Tabak, R.A. De Souza Penaloza and R.C. De Castro Miranda, Systemic risk measures. Physica A: Statistical Mechanics and its Applications, 2016, Vol. 442, pp. 329-342.

[17] R. Pasqualino, I. Monasterolo and A. Jones, An Integrated global food and energy security system dynamics model for addressing systemic risk. Sustainability, 2019, Vol. 11, 3995.

[18] J. Paulin, A. Calinescu And M. Wooldridge, Understanding flash crash contagion and systemic risk: A micro-macro agent-based approach. Journal of Economic Dynamics and Control, 2019, Vol. 100, pp. 200-229.

[19] S. Burgess, E. Fernandez-Corugedo, C. Groth, R. Harrison, F. Monti, K. Theodoridis and M. Waldron, The Bank of England's forecasting platform. Bank of England. 2013.

[20] R.A. Bradhurst, S.E. Roche, I.J. East, P. Kwan And M.G. Garner, A hybrid modeling approach to simulating foot-and-mouth disease outbreaks in Australian livestock. Frontiers in Environmental Science, 2015, Vol. 3, 17, https://doi.org/10.3389/fenvs.2015.00017.

[21] J. Boston, Anticipatory governance: how well is New Zealand safeguarding the future? Policy Quarterly, 2016, Vol. 12, No. 3, https://doi.org/10.26686/pq.v12i3.4614.

[22] K. Raworth, Doughnut economics: Seven ways to think like a 21st-Century Economist, Chelsea Green Publishing, London, 2018.

[23] R. Scholz and G. Steiner, The real type and ideal type of transdisciplinary processes: Part I - theoretical foundations. Sustainability Science, 2015, 10, pp. 527-544.

[24] N. Wognum, W.J.C. Verhagen, J. Stjepandić, Trans-disciplinary systems as complex systems, Advances in Transdisciplinary Engineering, 2017, Vol. 5, pp. 745-754.

[25] A.G. Haldane and R.M. May, Systemic risk in banking ecosystems. Nature, 2011, Vol. 469, pp. 351-355.

[26] D.E. Goldberg, The Design of Innovation: Lessons from and for Competent Genetic Algorithms, Dordrecht, Kluwer Academics Publishers, 2002.

[27] A. Biahmou et al., Towards agile enterprise rights management in engineering collaboration, International Journal of Agile Systems and Management, 2016, Vol. 9(4), pp. 302-325.

[28] R. Cont, A. Moussa and E.B. Santos, Network Structure and Systemic Risk in Banking Systems. In: J.P. Fouque and J.A. Langsam (eds.) Handbook on Systemic Risk, Cambridge University Press, Cambridge, 2013, pp. 327-368.

[29] N. Wognum, M. Wever, Stjepandić, J. Managing risks in knowledge exchange: Trade-offs and interdependencies, Advances in Transdisciplinary Engineering, 2016, Vol. 4, pp. 15-24.

[30] N. Wognum, C. Bil, F. Elgh, M. Peruzzini, J. Stjepandić, W.J.C. Verhagen, Transdisciplinary engineering research challenges, Advances in Transdisciplinary Engineering, Vol. 7, 2018, pp. 753-762. 